\title{
Self-heating property under ac magnetic field and its evaluation by ac/dc hysteresis loops of $\mathrm{NiFe}_{2} \mathrm{O}_{4}$ nanoparticles
}

\author{
H. Kobayashi, ${ }^{1, a)}$ A. Hirukawa, ${ }^{1}$ A. Tomitaka, ${ }^{1}$ T. Yamada, ${ }^{1}$ M. Jeun, ${ }^{2}$ S. Bae, ${ }^{2}$ and \\ Y. Takemura, a) \\ ${ }^{1}$ Department of Electrical and Computer Engineering, Yokohama National University, Yokohama 240-8501, \\ Japan \\ ${ }^{2}$ Biomagnetics Laboratory, Department of Electrical and Computer Engineering, National University \\ of Singapore, Singapore 117576, Singapore
}

(Presented 22 January 2010; received 30 October 2009; accepted 16 November 2009; published online 12 May 2010)

The temperature rise of $\mathrm{NiFe}_{2} \mathrm{O}_{4}$ nanoparticles having diameters of 242 and $7.7 \mathrm{~nm}$ was measured. The results of the measurement were analyzed by comparing the areas of the hysteresis loops in order to clarify the mechanism of heat dissipation in the samples. The hysteresis loops were obtained by applying both ac and dc magnetic fields. It was found that the contribution of magnetic relaxation losses to the heat dissipation was negligible in the case of $\mathrm{NiFe}_{2} \mathrm{O}_{4}$ nanoparticle of diameter $242 \mathrm{~nm}$. The contribution of the Neel relaxation to the heat dissipation in the case of $\mathrm{NiFe}_{2} \mathrm{O}_{4}$ nanoparticle of diameter $7.7 \mathrm{~nm}$ was observed as the difference between the areas of ac and dc hysteresis loops. From the dependences of temperature rise and hysteresis loops of the nanoparticles on the intensity and frequency of an applied magnetic field, the relaxation time for $\mathrm{NiFe}_{2} \mathrm{O}_{4}$ nanoparticle of diameter $7.7 \mathrm{~nm}$ was obtained as approximately $0.5-0.7 \mu \mathrm{s}$.

(C) 2010 American Institute of Physics. [doi:10.1063/1.3355936]

\section{INTRODUCTION}

Hyperthermia is a cancer treatment in which cancer cells are killed by heating. It is known that cells are killed at temperatures above $42.5^{\circ} \mathrm{C}$, furthermore, tumor cells are more sensitive to an increase in temperature than normal tissues because they have a weak cooling function. Magnetic nanoparticles can be used as heating agents for hyperthermia. ${ }^{1}$ It has been reported that the temperature of a tumor was increased by the self-heating of a magnetic nanoparticle injected into the tumor.

The self-heating property of magnetic nanoparticles is greatly dependent on their particle size. This property originates from hysteresis loss and magnetic relaxation loss. The heating mechanisms of magnetic nanoparticles have been elucidated by taking measurements of the magnetization curve and magnetic susceptibility under an ac magnetic field. ${ }^{2,3}$ However, there are few reports of ac magnetization curves measured at the high frequencies used for hyperthermia. ${ }^{4,5}$ In this study, the temperature rise and magnetization curves of $\mathrm{NiFe}_{2} \mathrm{O}_{4}$ nanoparticles were evaluated under both $\mathrm{dc}$ and ac fields. The magnetic relaxation time was derived from the field intensity and frequency dependences of the temperature rise and ac hysteresis.

\section{HEAT DISSIPATION OF MAGNETIC NANOPARTICLES}

Heat dissipation from magnetic particles is caused by hysteresis loss. It is principally related to pinning of magnetic domain walls at impurities and other factors.

\footnotetext{
${ }^{a)}$ Electronic addresses: d09gd135@ynu.ac.jp and takemura@ynu.ac.jp.
}

Other than the heating due to conventional hysteresis loss, heating also occurs as a result of magnetic relaxation losses, which are caused by the delay in the relaxation of the magnetic moment rotation. When the effective relaxation time of particles is longer than the period of an applied ac magnetic field, the particles are heated as the rotation of magnetic moment lags behind the changing applied magnetic field. The heating power due to the magnetic relaxation loss is given by the following equation ${ }^{6}$ :

$$
P=\pi \mu_{0} \chi_{0} H_{0}^{2} f \frac{2 \pi f \tau}{1+(2 \pi f \tau)^{2}},
$$

where $P$ is the heat dissipation value; $\mu_{0}$, the permeability of vacuum; $\chi_{0}$ the initial magnetic susceptibility; and $\tau$, the relaxation time. $H$ and $f$ are the intensity and frequency of an applied ac magnetic field, respectively.

\section{EXPERIMENTS}

$\mathrm{NiFe}_{2} \mathrm{O}_{4}$ nanoparticles of diameters $242 \pm 73$ and $7.7 \pm 1.0 \mathrm{~nm}$ were used as samples. The nanoparticles of diameters 242 and $7.7 \mathrm{~nm}$ were synthesized by a modified sol-gel method ${ }^{7}$ and by a high temperature thermal decom-
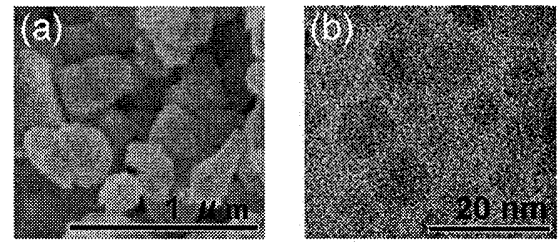

FIG. 1. Transmission electron microscopic images of $\mathrm{NiFe}_{2} \mathrm{O}_{4}$ nanoparticles of (a) diameter $242 \mathrm{~nm}$ and (b) diameter $7.7 \mathrm{~nm}$. 

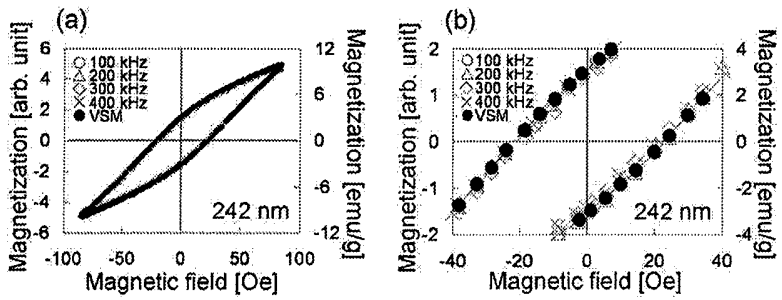

FIG. 2. (a) Minor hysteresis loops of the $\mathrm{NiFe}_{2} \mathrm{O}_{4}$ nanoparticle of diameter $242 \mathrm{~nm}$ measured under the applied dc and ac magnetic field of $60 \mathrm{Oe}$ at $100-400 \mathrm{kHz}$ and (b) its enlarged view.

position, respectively. Figures 1 (a) and $1(\mathrm{~b})$ show transmission electron microscopy (TEM) images of the nanoparticles. The particle size and its distribution were determined by the TEM images. The sampling frame size was $3 \mu \mathrm{m}^{2}$ and $600 \mathrm{~nm}^{2}$ for the $242 \mathrm{~nm}$ and $7.7 \mathrm{~nm}$ nanoparticles, respectively. The nanoparticle of diameter $242 \mathrm{~nm}$ exhibited ferromagnetic characteristic, whereas the nanoparticle of diameter $7.7 \mathrm{~nm}$ exhibited superparamagnetic characteristic. The nanoparticles were stored in a glass tube (inner diameter 1.0 $\mathrm{mm}$; length $5 \mathrm{~mm}$ ) in a dried powder state. The heating characteristics of the magnetic nanoparticles were investigated by measuring the temperature rise and the area of the ac/dc hysteresis loops. The dc hysteresis loop was measured by using a vibration sample magnetometer. The temperature rise and ac hysteresis loop were measured under an ac magnetic field of 30-210 Oe (rms) with a frequency of $50-400 \mathrm{kHz}$. The temperature was measured by using an optical fiber sensor.

\section{RESULTS AND DISCUSSION}

\section{A. Frequency dependence}

The minor loops of the hysteresis of the samples that were measured under $\mathrm{dc}$ and ac $(100-400 \mathrm{kHz})$ magnetic fields are shown in Figs. 2 and 3. The field intensity was 60 Oe rms for ac and 85 Oe for the dc field, respectively. The minor loop in the case of the sample with nanoparticles of diameter $242 \mathrm{~nm}$ was almost constant for both $\mathrm{dc}$ and ac fields of various frequency, as indicated by Figs. 2(a) and 2(b). On the other hand, the minor loops of the sample with nanoparticles of diameter $7.7 \mathrm{~nm}$ were dependent on the field frequency, as indicated in Fig. 3(b). The coercivity for the minor loop increased with increasing field frequency. This occurred because the rotation of magnetization lags behind the changing applied magnetic field. The areas of the minor loops were calculated and are shown in Figs. 4(a) and 4(b). The slope of the initial temperature rise obtained from the results of temperature measurement (not shown) is also plotted in Figs. 4(a) and 4(b), respectively. The slope was divided by the applied frequency in order to compare it with the hysteresis area. As indicated by Fig. 4(a), the area of the minor loop and the heat dissipation normalized by frequency of the $\mathrm{NiFe}_{2} \mathrm{O}_{4}$ nanoparticle of diameter $242 \mathrm{~nm}$ were not dependent on the applied field frequency. The dotted line in the figure represents a constant value for the area of hysteresis and the slope. This indicates that the self-heating of the sample is due to hysteresis loss, as observed for even dc field excitation, and that there is no contribution from relaxation
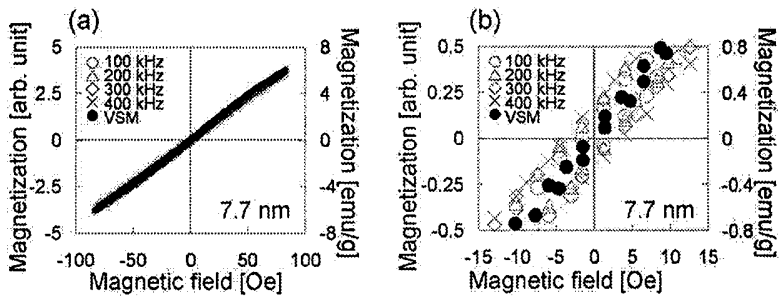

FIG. 3. (a) Minor hysteresis loops of the $\mathrm{NiFe}_{2} \mathrm{O}_{4}$ nanoparticle of diameter $7.7 \mathrm{~nm}$ measured under the applied $\mathrm{dc}$ and ac magnetic field of $60 \mathrm{Oe}$ at $100-400 \mathrm{kHz}$ and (b) its enlarged view.

losses. In the case of the sample with nanoparticles having diameter $7.7 \mathrm{~nm}$, the area of hysteresis and the slope of temperature rise were in good agreement. However, in this case, they exhibited frequency dependence, this suggests that the self-heating of the $\mathrm{NiFe}_{2} \mathrm{O}_{4}$ nanoparticles having diameter $7.7 \mathrm{~nm}$ was partially due to magnetic relaxation loss.

\section{B. Field intensity dependence}

The dependences of heat dissipation on the intensity of the applied field are shown in Figs. 5(a) and 5(b) for the samples with nanoparticles having diameters $242 \mathrm{~nm}$ and 7.7 $\mathrm{nm}$, respectively. The area of the ac minor loop and the slope of temperature rise measured by the applied field at $100 \mathrm{kHz}$ are also plotted, along with the area of the dc hysteresis loop. The sample with 242-nm-diameter nanoparticles had the same areas for dc and ac hysteresis loops, and its field intensity dependence agreed with the temperature rise. In the case

(a)

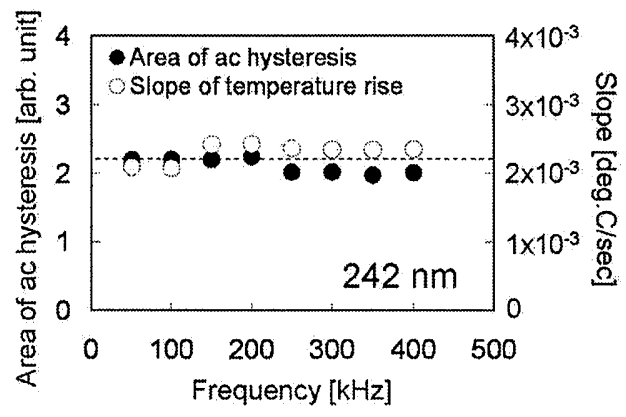

(b)

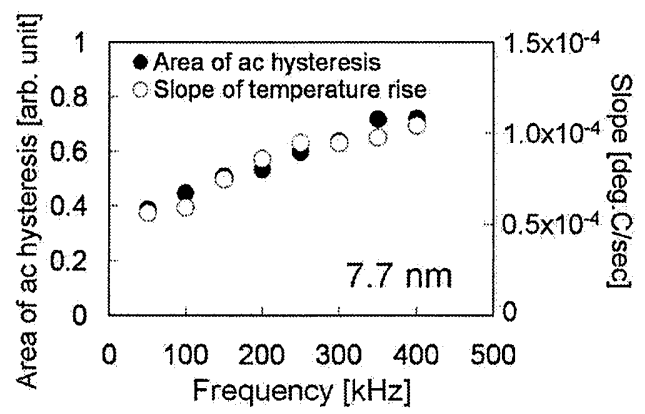

FIG. 4. Frequency dependences of the area of ac hysteresis loop and slope of the temperature rise of the $\mathrm{NiFe}_{2} \mathrm{O}_{4}$ nanoparticles with (a) diameter 242 $\mathrm{nm}$ and (b) diameter $7.7 \mathrm{~nm}$ measured under the applied ac magnetic field of $60 \mathrm{Oe}$. 
(a)

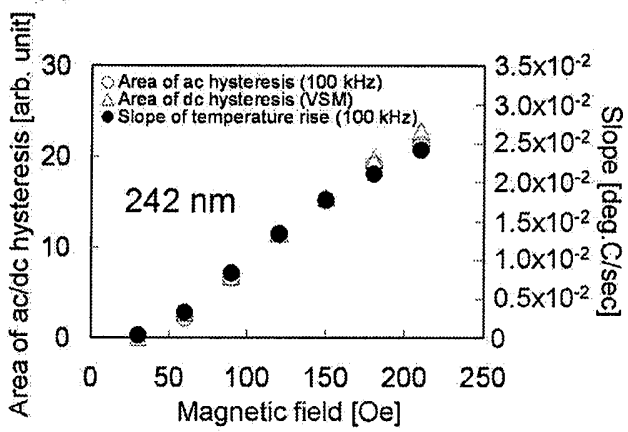

(b)

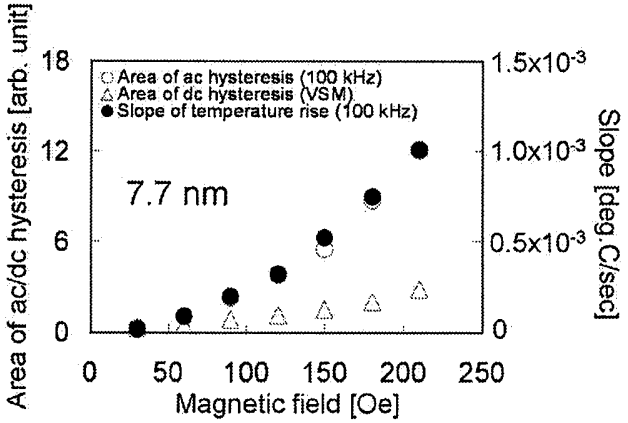

FIG. 5. Field intensity dependences of the area of ac/dc hysteresis loop and slope of the temperature rise of the $\mathrm{NiFe}_{2} \mathrm{O}_{4}$ nanoparticles with (a) diameter $242 \mathrm{~nm}$ and (b) diameter $7.7 \mathrm{~nm}$. The frequency of the ac field was $100 \mathrm{kHz}$ for the measurement of ac hysteresis and temperature rise.

of the sample with 7.7-nm-diameter nanoparticles, it was confirmed that the area of the ac hysteresis agreed with the slope of the temperature rise; however, the area of the dc hysteresis loop was smaller than that of ac hysteresis. This difference between ac and dc hysteresis loops is dominantly attributed to the Neel relaxation, because the Brownian relaxation was negligible for the samples in the condensed powder state.

The power dissipation described by Eq. (1) and its experimental result including field and frequency dependence could give the relaxation time $\tau$ without determining a mag- netic susceptibility $\chi_{0}$. From the results shown in Figs. 4(b) and $5(\mathrm{~b})$, the relaxation time for the $\mathrm{NiFe}_{2} \mathrm{O}_{4}$ nanoparticle of diameter $7.7 \mathrm{~nm}$ was determined to be approximately 0.5-0.7 $\mu$ s. If an ac magnetic field with a frequency of approximately $1 \mathrm{MHz}$, which closely matches the relaxation time, is applied to this sample, a higher temperature rise is expected as a resonant condition of the magnetization.

\section{CONCLUSION}

Self-heating properties of the $\mathrm{NiFe}_{2} \mathrm{O}_{4}$ nanoparticles of diameters of 242 and $7.7 \mathrm{~nm}$ were studied. The dependences of their temperature rise on the intensity and frequency of an applied ac magnetic field agreed well with the corresponding dependences of the areas of the hysteresis loops. The temperature rise characteristic and the area of the ac hysteresis loop of the nanoparticles with diameter $242 \mathrm{~nm}$ did not depend on the field frequency, which indicated that the sample did not exhibit magnetic relaxation losses. By comparing the areas of ac and dc hysteresis loops, the contribution of the Néel relaxation to the heat dissipation could be clarified for the sample with nanoparticles having diameter $7.7 \mathrm{~nm}$. The relaxation time of approximately $0.5-0.7 \mu$ s was suggested for this superparamagnetic nanoparticle. These analyses on contribution of the Néel relaxation and relaxation time are significant for developing thermal therapy of cancer using the magnetic nanoparticles, because optimum conditions of the nanoparticles and an applied magnetic field frequency can be examined.

'Q. A. Pankhurst, J. Connolly, S. K. Jones, and J. Donson, J. Phys. D 36, R167 (2003).

${ }^{2}$ R. Müller, R. Hergt, M. Zeisberger, and W. Gawalek, J. Magn. Magn. Mater. 289, 13 (2005)

${ }^{3}$ S. Dutz, R. Hergt, J. Mürbe, R. Müller, M. Zeisberger, W. Andrä, J. Töpfer, and M. E. Bellemann, J. Magn. Magn. Mater. 308, 305 (2007).

${ }^{4}$ A. K. Giri, K. M. Chowdary, and S. A. Majetich, Mater. Phys. Mech. 1, 1 (2000).

${ }^{5}$ B. E. Kashevsky, V. E. Agabelov, S. Kashevsky, K. A. Kekalo, E. Y. Manina, I. V. Prokhorov, and V. S. Ulashchik, China Particuol. 6, 322 (2008).

${ }^{6}$ R. E. Rosensweig, J. Magn. Magn. Mater. 252, 370 (2002).

${ }^{7}$ S. W. Lee, S. Bae, Y. Takemura, E. Yamashita, J. Kunisaki, S. Zurn, and C. S. Kim, IEEE Trans. Magn. 42, 2833 (2006). 